\title{
Neck Grasp Predicts Obstructive Sleep Apnea in Type 2 Diabetes Mellitus
}

\author{
P. J. Edmonds $\mathbb{D}^{1},{ }^{1}$ K. Gunasekaran $\left(\mathbb{D},{ }^{2}\right.$ and L. C. Edmonds ${ }^{3}$ \\ ${ }^{1}$ Vanderbilt University Medical Center, Department of Medicine, 1161 21st Avenue South, Nashville, TN 37232, USA \\ ${ }^{2}$ Yale New Haven Health, Bridgeport Hospital, Pulmonary Medicine, 267 Grant Street, Bridgeport, CT 06610, USA \\ ${ }^{3}$ Sleep Disorder Center, Research Institute, Bassett Medical Center, 1 Atwell Road, Cooperstown, NY, USA \\ Correspondence should be addressed to P. J. Edmonds; peter.j.edmonds@vumc.org
}

Received 4 March 2019; Accepted 7 June 2019; Published 1 July 2019

Academic Editor: Marco Zucconi

Copyright (C) 2019 P. J. Edmonds et al. This is an open access article distributed under the Creative Commons Attribution License, which permits unrestricted use, distribution, and reproduction in any medium, provided the original work is properly cited.

\begin{abstract}
Aims. Obstructive sleep apnea (OSA) is a common disorder with high morbidity, mortality, and an increasing prevalence in the general population. It has an even higher prevalence among individuals with type 2 diabetes mellitus (DM). The snoring, tiredness, observed apnea, high blood pressure, body-mass-index, age, neck circumference and male gender (STOP-BANG) questionnaire and Berlin Questionnaire can be cumbersome in clinical practice and require subjective data on sleepiness. We proposed prospectively studying a primary care population with type $2 \mathrm{DM}$ comparing neck grasp, neck circumference, and common screening questionnaires to identify OSA. Methods. Persons with a diagnosis of type 2 DM were recruited from a primary care clinic. Participants were screened using Easy Sleep Apnea Predictor (ESAP), STOP-Bang questionnaire, and Berlin questionnaire. A positive ESAP was defined as a $1 \mathrm{~cm}$ gap when a patient encircled their hands around the neck. All subjects underwent in-laboratory PSG testing. Results. Forty-three participants were enrolled and the prevalence of OSA was 90.7\% (AHI $\geq 5$ ). The median BMI was 38.0. The prevalence of mild OSA by PSG (AHI 5-14) was 27.9\%, moderate OSA (AHI 15-29) was $25.6 \%$, and severe OSA (AHI $>30$ ) was $37.2 \%$. For mild OSA both ESAP and neck circumference showed $100 \%$ specificity. Conclusions. This study reinforces the need for screening diabetic persons for obstructive sleep apnea. ESAP and neck circumference are useful for identifying persons with type $2 \mathrm{DM}$ who are at risk for OSA. Together these findings could improve recognition of OSA in persons at risk for cardiovascular disease. Trial Registration of "Neck grasp as a predictor of Sleep Apnea," https://clinicaltrials.gov/ct2/show/NCT02474823, Clinical Trials.gov Identifier, is NCT02474823.
\end{abstract}

\section{Introduction}

Obstructive sleep apnea (OSA) is a common disorder with high morbidity, mortality, and increasing prevalence [1]. Moreover, despite efforts to improve recognition, $80 \%$ remains undiagnosed [2]. In the general population obstructive sleep apnea affects 3-7\% of persons [3]. Persons with type 2 diabetes mellitus (DM) are at increased risk for OSA with a reported prevalence ranging from $23-90 \%$ [4-6]. OSA is an independent risk factor for type $2 \mathrm{DM}$ [7]. Both OSA and type $2 \mathrm{DM}$ are strong risk factors for cardiovascular disease [3, 810].

While several questionnaires and models have been validated for the clinical prediction of sleep apnea, few have been validated in a population of type 2 diabetes mellitus [11-13].
The snoring, tiredness, observed apnea, high blood pressure, body-mass-index, age, neck circumference and male gender (STOP-Bang) questionnaire and Berlin Questionnaire (BQ) [14] can be cumbersome in clinical practice and require subjective data on sleepiness and third party reporting of snoring $[11,14]$. A simple, reliable, and objective screening test could improve a timely diagnosis of OSA in persons with type 2 DM.

Neck circumference has a well-studied relationship with OSA. Decreased cross sectional area, increased compliance, and mass loading of the upper airway all contribute to sleep disordered breathing [15]. Guidelines suggest screening for OSA with a collar size greater than 17 inches in males and 16 inches in females [16]. A previous study of neck grasp (easy sleep apnea predictor, ESAP) for identifying mild OSA 
TABLE 1: Patient demographics and characteristics.

\begin{tabular}{|c|c|}
\hline Characteristic & $\mathrm{N}(\%)$ \\
\hline Male & $20(46.51)$ \\
\hline Female & $23(53.49$ \\
\hline \multicolumn{2}{|c|}{$\mathrm{AHI}^{\dagger}$ group (by PSG) } \\
\hline Positive $(\mathrm{AHI}>5)$ & $39(90.7)$ \\
\hline Mild (AHI 5-14) & $12(27.91)$ \\
\hline Moderate (AHI 15-29 & $11(25.58)$ \\
\hline Severe $(\mathrm{AHI} \geq 30)$ & $16(37.21)$ \\
\hline $\mathrm{BMI}^{\S} \leq 35$ & $15(34.88)$ \\
\hline $\mathrm{BMI}>35$ & $28(65.12)$ \\
\hline Positive Neck Circumference* & $19(44.19)$ \\
\hline Negative Neck Circumference & $24(55.81)$ \\
\hline \multicolumn{2}{|c|}{ Mean (+/-SD, range) } \\
\hline AHI & $\begin{array}{c}31.24(\mathrm{SD}+/-28.14 \\
1-117) \\
\end{array}$ \\
\hline $\mathrm{BMI}\left(\mathrm{kg} / \mathrm{m}^{2}\right)$ & $\begin{array}{c}38.26(\mathrm{SD}+/-7.69 \\
24-55)\end{array}$ \\
\hline Neck Circumference $(\mathrm{cm})$ & $\begin{array}{c}41.64(\mathrm{SD}+/-4.04 \\
33-51)\end{array}$ \\
\hline $\begin{array}{l}\text { Gap measure }(\mathrm{cm} ; \text { ESAP } \\
\text { negative }=0 \mathrm{~cm})\end{array}$ & $3.20(\mathrm{SD}+/-3.81,0-13)$ \\
\hline \multicolumn{2}{|c|}{$\begin{array}{l}\dagger \text { AHI (apnea-hypopnea index is the number of apneas and hypopneas per } \\
\text { hour of recording. This was scored using type } 1 \text { polysomnogram with AASM } \\
2016 \text { scoring rules, specifically hypopnea rule of } 3 \% \text { or arousal. } \\
\S \text { BMI (body mass index) is the weight in kilograms divided by the square of } \\
\text { the height in meters. } \\
\text { g Gap measure was the number of } \mathrm{cm} \text { between the fingers in a patient with } \\
\text { a positive ESAP. } \\
\text { * A positive neck circumference was }>16 \text { inches in females and }>17 \text { inches } \\
\text { in males. A negative neck circumference was }<16 \text { inches in women and }<17 \\
\text { inches in men. }\end{array}$} \\
\hline
\end{tabular}

(AHI $>5$ ) reported a sensitivity of $68.3 \%$ and a specificity of $100 \%$ in a symptomatic sleep clinic population [17].

We proposed to prospectively study the usefulness of the simple neck grasp, neck circumference, BMI (body mass index) and common screening questionnaires to identify OSA in type 2 diabetes mellitus. We hypothesized that a positive ESAP is noninferior to Stop-Bang, Berlin Questionnaire at recognizing OSA in type $2 \mathrm{DM}$.

\section{Methods}

The institutional review board approved the study design involving noninvasive procedure with human subjects. The approved protocol (IRB number 2012) included informed signed consent from all participants and oversight to ensure operation within the approved protocol. Adults (age 18 years and older) with a diagnosis of type $2 \mathrm{DM}$ were serially recruited from a general internal medicine clinic at routine health visits from 2015-2016. Exclusion criteria included a known diagnosis of OSA, age $<18$, unstable cardiopulmonary disease, inability to perform the neck grasp or complete questionnaires. Participants were screened using the ESAP (easy sleep apnea predictor), STOP-BANG questionnaire, neck circumference $(>16$ inches in females and $>17$ inches in males) [16] and Berlin questionnaire. A positive ESAP was defined as a $1 \mathrm{~cm}$ gap when patient encircled their hands around the neck (see Figure 1). All subjects underwent inlaboratory Type 1 polysomnogram (PSG). Type 1 polysomnogram was scored using AASM 2016 scoring rules, specifically hypopnea rule of $3 \%$ or arousal. Participants were reimbursed 50 dollars for participation.

2.1. Statistical Analysis. Demographic variables between groups were compared using Chi-squared and the $t$ test (BMI, age, AHI). Data are presented as mean \pm standard deviation. Using AHI from polysomnogram as the gold standard for OSA diagnosis the sensitivity (SN), specificity (SP), positive predictive value (PPV), and negative predictive value (NPV) to predict OSA were calculated. Sample size calculation used prevalence of OSA ( $7 \%$ in the general population and estimated $20 \%$ in diabetes mellitus) type 1 error (0.05) and power (0.8). Continuous variables (BMI, neck circumference, and ESAP gap measure) were compared to AHI using the receiver operator curve to calculate sensitivity $(\mathrm{SN})$, specificity (SP), and area under the curve (AUC). An AUC of $\geq 0.9$ indicated outstanding discrimination, $0.8 \leq$ AUC $<0.9$ indicated good discrimination, $0.7 \leq \mathrm{ACU}<0.8$ indicated acceptable discrimination, and AUC $=0.5$ indicated no discrimination.

\section{Results}

3.1. Study Population Characteristics. Eight-six participants were screened in a primary care clinic. Forty-three persons met inclusion criteria (53\% female, Table 1). The most common reasons for exclusion were prior diagnosis of OSA or not having diabetes mellitus. The prevalence of OSA was 90.7\%. The median BMI was 38.0. The prevalence of mild OSA by PSG (AHI 5-14) was 27.9\%, moderate OSA (AHI 1529 ) was $25.6 \%$, and severe OSA (AHI $>30$ ) was $37.2 \%$. In the study population $11.63 \%$ of the subjects were uncertain if they snored. The neck circumference was increased $(>43.2 \mathrm{~cm}$ in males and $>40.6 \mathrm{~cm}$ in females) in $44 \%$ of the participants. As expected in a population of type $2 \mathrm{DM}$, the BMI was $\geq 35$ in a majority of subjects $(65.12 \%)$.

3.2. Validation of Screening Tests for Mild, Moderate, and Severe OSA. For Mild OSA (AHI 5-14) the most sensitive screening test was STOP-Bang score $\geq 3$ with a sensitivity of $87.2 \%$. The most specific screening tests were ESAP and neck circumference both with $100 \%$ specificity (Table 2). For moderate OSA (AHI 15-29) the most sensitive screening test was the Berlin high-risk group. The most specific screening test was neck circumference with a specificity of $75 \%$ (Table 2 ). The most sensitive screening tests for Severe OSA (AHI $\geq 30$ ) were STOP-Bang and the Berlin Questionnaire both with a sensitivity of $93.8 \%$. The most specific screening test was neck circumference with a specificity of $70.4 \%$ (Table 2 ).

3.3. Linear Regression of Continuous Variables. Continuous variables (BMI, neck circumference, and ESAP gap measure) 


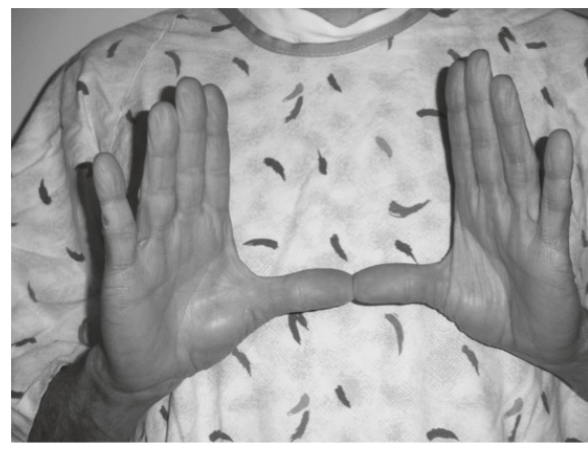

(a)

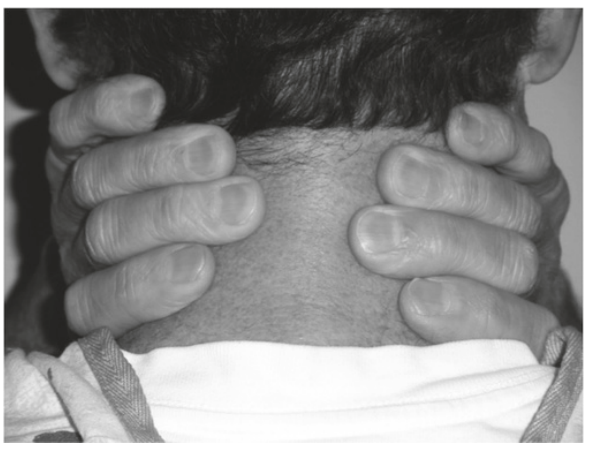

(b)

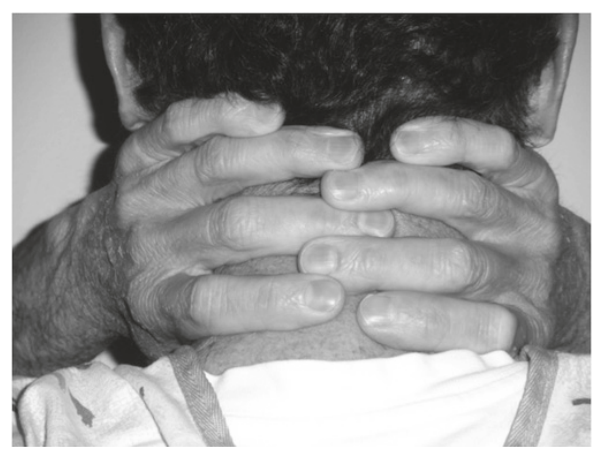

(c)

FIGURE 1: (a) Easy sleep apnea predictor (ESAP) entails placing both thumbs together at the anterior of the neck and encircling the fingers in the posterior. (b) Positive ESAP is shown. The patient is unable to encircle their neck resulting in at least a $1 \mathrm{~cm}$ gap between fingertips. (c) Negative ESAP is shown. The patient is able to encircle their neck defined as touching fingertips in the posterior neck. Figures reproduced with author permission [17].

TABLE 2: Comparison of for screening tests for sensitivity, specificity, positive predictive value, and negative predictive value for mild OSA, moderate, and severe OSA.

\begin{tabular}{|c|c|c|c|c|}
\hline \multicolumn{5}{|c|}{ Mild OSA (AHI 5-14, $\mathrm{n}=12$ ) } \\
\hline Screening Test & Sensitivity & Specificity & Positive Predictive Value & Negative Predictive Value \\
\hline Positive ESAP $*$ & 55 & 100 & 100 & 18.2 \\
\hline STOP-Bang $\geq 3$ & 87.2 & 0 & 89.5 & 0 \\
\hline Berlin High Risk $^{\dagger}$ & 79.5 & 0 & 88.6 & 0 \\
\hline $\mathrm{BMI} \geq 35$ & 69.2 & 75 & 96.4 & 20 \\
\hline Positive Neck Circumference ${ }^{\S}$ & 48.7 & 100 & 100 & 16.7 \\
\hline \multicolumn{5}{|c|}{ Moderate OSA (AHI 15-29, n=11) } \\
\hline Positive ESAP & 60.7 & 68.8 & 77.3 & 50 \\
\hline STOP-Bang $\geq 3$ & 92.6 & 18.8 & 65.8 & 60 \\
\hline Berlin High Risk & 88.9 & 31.3 & 68.6 & 62.5 \\
\hline $\mathrm{BMI} \geq 35$ & 74.1 & 50 & 71.4 & 53.3 \\
\hline Positive Neck Circumference & 55.6 & 75 & 78.9 & 50 \\
\hline \multicolumn{5}{|c|}{ Severe OSA (AHI >30, $\mathrm{n}=16)$} \\
\hline Positive ESAP & 62.5 & 57.1 & 45.5 & 72.7 \\
\hline Stop-Bang $\geq 3$ & 93.8 & 14.8 & 39.5 & 80 \\
\hline Berlin High Risk & 93.8 & 25.9 & 42.9 & 87.5 \\
\hline $\mathrm{BMI} \geq 35$ & 75 & 40.7 & 42.9 & 73.3 \\
\hline Neck Circumference & 68.8 & 70.4 & 57.9 & 79.2 \\
\hline
\end{tabular}

* A positive ESAP (easy sleep apnea predictor) is defined as the failure to encircle the neck using hand grasp with a 1cm gap between fingers (Figure 1).

${ }^{\dagger}$ High-risk berlin questionnaire is 2 or more positive categories on the screening questionnaire.

${ }^{\varsigma} \mathrm{A}$ positive neck circumference was $>16$ inches in females and $>17$ inches in males. 
TABLE 3: Comparison of Linear regression (c-statistic) for ESAP, $\mathrm{BMI}$, neck circumference, and gap measure.

\begin{tabular}{lc}
\hline Screening Test & Area under the curve \\
\hline ESAP & 0.657 \\
\hline BMI (continuous) & 0.705 \\
\hline Neck Circumference $(\mathrm{cm})$ & 0.885 \\
\hline Gap Measure $*(\mathrm{~cm})$ & 0.724 \\
\hline
\end{tabular}

${ }^{*}$ Gap measure was the number of $\mathrm{cm}$ between the fingers in a patient with a positive ESAP. STOP-Bang and BQ were not included because they were scored dichotomously.

were compared to AHI (Table 3). In participants with a positive ESAP, the number of centimeters between the fingers was used as a gap measure. Area under the recover operator curve was highest for neck circumference (0.885) followed by ESAP gap measure (0.724), and BMI (0.705) (Table 3). STOPBang and BQ were not included because they were scored dichotomously.

\section{Discussion}

In this study, we demonstrate that neck circumference performs well as a clinical screening tool in the diabetic population. Furthermore, we show that the ESAP neck grasp is a simple alternative to measuring neck circumference. Participants with type 2 DM have a high prevalence of obstructive sleep apnea, which is consistent with prior reports [4-6].

Our key findings are the usefulness of neck girth based predictors (neck circumference and ESAP) of OSA in type 2 DM. With the high specificity and positive predictive value, ESAP is reliable at recognizing mild disease $(\mathrm{AHI} \geq 5)$. The sensitivity, specificity and area under the curve for neck circumference and ESAP showed similar results (100\% specificity for mild OSA), with neck circumference showing slight superiority. One advantage of ESAP compared to neck circumference is that gender normative standards need not be applied ( $>17$ inches in males and $>16$ inches in females) [16]. Hand to neck size is individually normative. The lower sensitivity of neck circumference and ESAP compared to STOP-Bang and BQ questionnaires could be attributed to craniofacial abnormalities, tonsillar or adenoidal enlargement not assessed by neck girth alone. Unlike STOP-BANG and the Berlin Questionnaire, ESAP requires no other information or point assignment to predict OSA $(A H I \geq 5)$ in type 2 diabetics. In addition to simplicity, relying on neck girth avoids subjective questioning. Snoring status, an important component of STOP-Bang and BQ, was uncertain in $11.6 \%$ of study participants.

We confirmed prior findings that neck circumference is a better predictor than BMI alone [18] and that guidelines using neck circumference remain useful [19-21]. STOP-BANG has similar sensitivity and specificity compared to prior studies in surgical and primary care patients [11, 14, 22]. Berlin Questionnaire also showed similar sensitivity and specificity to prior validation [23]. A negative ESAP or a normal neck size does not exclude OSA because of the low sensitivity.
However, a positive test correlates well with persons who are at risk for OSA.

As continuous variables, ESAP and neck circumference did not reliably predict severity of OSA, nor did BMI. As a noncontinuous variable BMI also lacked the specificity seen in neck girth based tests. The neck-based predictors likely see the increase specificity over BMI due to anatomical role of mass loading on the upper airway in OSA.

The relationship between OSA and type 2 DM was originally attributed to obesity, but further research has shown OSA is an independent risk factor for type 2 DM [7]. OSA affects physiologic and hormonal pathways including hypoxia induced changes in glucose metabolism [24], sleep fragmentation affecting hypothalamic-pituitary-adrenal axis [25], and increased levels of IL-6, TNF-alpha, adiponectin, and MCP-1 [26-28]. Numerous studies show an association between sleep apnea and cardiovascular disease [29], glucose metabolism [30], and systemic hypertension [31]. OSA is an independent risk factor for all-cause mortality after controlling for obesity and cardiovascular disease [3, 8-10]. Guidelines from the International Diabetes Federation and the American Diabetes Association recommend screening all individuals with type 2 DM for OSA [19, 32].

The strengths of this study include using a prospective study design that enrolled participants who were not presenting with sleep complaints. By using in lab polysomnography instead of HST as the gold standard false negatives or miss classifications are minimized. In addition to simplicity, relying on neck girth avoids subjective questioning. Snoring status, an important component of STOP-Bang and BQ, was uncertain in $11.6 \%$ of study participants.

Limitations of this study include the small sample size and the higher than anticipated prevalence of OSA in the population. Areas of further research include testing if our findings would be useful in other similarly obese populations, such as bariatric surgery patients. Neck grasp is unstudied in truck drivers and department of transport examinations, a population where it might be useful to avoid historical bias against reporting snoring and sleepiness.

In summary, this study reinforces the importance of screening for obstructive sleep apnea in persons with type 2 DM. ESAP and neck circumference are useful for identifying individuals with DM2 who are at risk for mild OSA. A negative ESAP or normal neck circumference does not exclude OSA. Together these findings could improve recognition of OSA in individuals at risk for cardiovascular disease.

\section{Data Availability}

The data used to support the findings of this study are available from the corresponding author upon request.

\section{Disclosure}

This manuscript has not been published and is not under review for submission at any other journals. This data was presented as poster in June of 2016. The funder was not 
involved in study design, data collection, data analysis, manuscript preparation, or publication decisions.

\section{Conflicts of Interest}

The authors declare that there are no conflicts of interest regarding the publication of this paper.

\section{Authors' Contributions}

All authors made significant contribution to the research in the manuscript and approve of its claims.

\section{Acknowledgments}

Catherine Gilmore and Jennifer Victory contributed to this study through data collection and technical support. This study was funded in part by the E Donnell Thomas research foundation for graduate medical education. Funding provided reimbursement of study participants.

\section{References}

[1] P. Jennum, P. Tønnesen, R. Ibsen, and J. Kjellberg, "All-cause mortality from obstructive sleep apnea in male and female patients with and without continuous positive airway pressure treatment: a registry study with 10 years of follow-up," Nature and Science of Sleep, vol. 7, pp. 43-50, 2015.

[2] P. E. Peppard, T. Young, J. H. Barnet, M. Palta, E. W. Hagen, and K. M. Hla, "Increased prevalence of sleep-disordered breathing in adults," American Journal of Epidemiology, vol. 177, no. 9, pp. 1006-1014, 2013.

[3] N. M. Punjabi, B. S. Caffo, J. L. Goodwin et al., "Sleep-disordered breathing and mortality: a prospective cohort study," PLoS Medicine, vol. 6, no. 8, Article ID 1000132, 2009.

[4] G. D. Foster, M. H. Sanders, R. Millman et al., "Obstructive sleep apnea among obese patients with type 2 diabetes," Diabetes Care, vol. 32, no. 6, pp. 1017-1019, 2009.

[5] K. Mahmood, N. Akhter, K. Eldeirawi et al., "Prevalence of type 2 diabetes in patients with obstructive sleep apnea in a multiethnic sample," Journal of Clinical Sleep Medicine, vol. 5, no. 3, pp. 215-221, 2009.

[6] S. D. West, D. J. Nicoll, and J. R. Stradling, "Prevalence of obstructive sleep apnoea in men with type 2 diabetes," Thorax, vol. 61, no. 11, pp. 945-950, 2006.

[7] N. Botros, J. Concato, V. Mohsenin, B. Selim, K. Doctor, and H. K. Yaggi, "Obstructive sleep apnea as a risk factor for type 2 diabetes," American Journal of Medicine, vol. 122, no. 12, pp. 1122-1127, 2009.

[8] N. S. Marshall, K. K. H. Wong, P. Y. Liu, S. R. J. Cullen, M. W. Knuiman, and R. R. Grunstein, "Sleep apnea as an independent risk factor for all-cause mortality: the Busselton Health Study," SLEEP, vol. 31, no. 8, pp. 1079-1085, 2008.

[9] H. K. Yaggi, J. Concato, W. N. Kernan, J. H. Lichtman, L. M. Brass, and V. Mohsenin, "Obstructive sleep apnea as a risk factor for stroke and death," The New England Journal of Medicine, vol. 353, no. 19, pp. 2034-2041, 2005.

[10] T. Young, L. Finn, P. E. Peppard et al., "Sleep disordered breathing and mortality: eighteen-year follow-up of the wisconsin sleep cohort," SLEEP, vol. 31, no. 8, pp. 1071-1078, 2008.
[11] F. Chung, H. R. Abdullah, and P. Liao, "Stop-bang questionnaire: a practical approach to screen for obstructive sleep apnea," CHEST, vol. 149, no. 3, pp. 631-638, 2016.

[12] K. Westlake and J. Polak, "Screening for obstructive sleep apnea in type 2 diabetes patients - questionnaires are not good enough," Frontiers in Endocrinology, vol. 7, no. 124, 2016.

[13] A. Pataka, G. Kalamaras, E. Daskalopoulou, and P. Argyropoulou, "Sleep questionnaires for the screening of obstructive sleep apnea in patients with type 2 diabetes mellitus compared with non-diabetic patients," Journal of Diabetes, 2018.

[14] F. Chung, B. Yegneswaran, P. Liao et al., "Validation of the Berlin questionnaire and American Society of Anesthesiologists checklist as screening tools for obstructive sleep apnea in surgical patients," Anesthesiology, vol. 108, no. 5, pp. 822-830, 2008.

[15] J. S. Koenig and B. T. Thach, "Effects of mass loading on the upper airway," Journal of Applied Physiology, vol. 64, no. 6, pp. 2294-2299, 1988.

[16] L. J. Epstein, D. Kristo, P. J. Strollo Jr. et al., "Clinical guideline for the evaluation, management and long-term care of obstructive sleep apnea in adults," Journal of Clinical Sleep Medicine, vol. 5, no. 3, pp. 263-276, 2009.

[17] P. J. Edmonds and L. C. Edmonds, "A pilot study of the inability to fit hands around neck as a predictor of obstructive sleep apnea," North American Journal of Medical Sciences, vol. 7, no. 12, pp. 553-557, 2015.

[18] V. Hoffstein and S. Mateika, "Differences in abdominal and neck circumferences in patients with and without obstructive sleep apnoea," European Respiratory Journal, vol. 5, no. 4, pp. 377-381, 1992.

[19] American Diabetes Association (ADA), "Standards of medical care in diabetes," Diabetes Care, vol. 37, pp. S14-S80, 2014.

[20] R. J. Davies and J. R. Stradling, “The relationship between neck circumference, radiographic pharyngeal anatomy, and the obstructive sleep apnoea syndrome," European Respiratory Journal, vol. 3, no. 5, pp. 509-514, 1990.

[21] I. Katz, J. Stradling, A. S. Slutsky, N. Zamel, and V. Hoffstein, "Do patients with obstructive sleep apnea have thick necks?" American Review of Respiratory Disease, vol. 141, no. 5 I, pp. 1228-1231, 1990.

[22] G. E. Silva, K. D. Vana, J. L. Goodwin, D. L. Sherrill, and S. F. Quan, "Identification of patients with sleep disordered breathing: comparing the four-variable screening tool, STOP, STOP-bang, and epworth sleepiness scales," Journal of Clinical Sleep Medicine, vol. 7, no. 5, pp. 467-472, 2011.

[23] N. C. Netzer, R. A. Stoohs, C. M. Netzer, K. Clark, and K. P. Strohl, "Using the berlin questionnaire to identify patients at risk for the sleep apnea syndrome," Annals of Internal Medicine, vol. 131, no. 7, pp. 485-491, 1999.

[24] V. Y. Polotsky, J. Li, N. M. Punjabi et al., "Intermittent hypoxia increases insulin resistance in genetically obese mice," The Journal of Physiology, vol. 552, no. 1, pp. 253-264, 2003.

[25] M. Ekstedt, T. Åkerstedt, and M. Söderström, "Microarousals during sleep are associated with increased levels of lipids, cortisol, and blood pressure," Psychosomatic Medicine, vol. 66, no. 6, pp. 925-931, 2004.

[26] S. Imagawa, Y. Yamaguchi, K. Ogawa et al., "Interleukin-6 and tumor necrosis factor-alpha in patients with obstructive sleep apnea-hypopnea syndrome," Respiration, vol. 71, no. 1, pp. 24$29,2004$. 
[27] J. Kim, C. H. Lee, C. S. Park, B. G. Kim, S. W. Kim, and J. H. Cho, "Plasma levels of MCP-1 and adiponectin in obstructive sleep apnea syndrome," JAMA Otolaryngology-Head \& Neck Surgery, vol. 136, no. 9, pp. 896-899, 2010.

[28] H. Lui, J. Lui, S. Xiong et al., "The change of interleukin-6 and tumor necrosis factor in patients with obstructive sleep apnea syndrome," Journal of Tongji Medical University, vol. 20, no. 3, pp. 200-202, 2000.

[29] D. Sorajja, A. S. Gami, V. K. Somers, T. R. Behrenbeck, A. Garcia-Touchard, and F. Lopez-Jimenez, "Independent association between obstructive sleep apnea and subclinical coronary artery disease," CHEST, vol. 133, no. 4, pp. 927-933, 2008.

[30] N. M. Punjabi and V. Y. Polotsky, "Disorders of glucose metabolism in sleep apnea," Journal of Applied Physiology, vol. 99, no. 5, pp. 1998-2007, 2005.

[31] P. E. Peppard, T. Young, M. Palta, and J. Skatrud, "Prospective study of the association between sleep-disordered breathing and hypertension," The New England Journal of Medicine, vol. 342, no. 19, pp. 1378-1384, 2000.

[32] IDF, The IDF Consensus Statement on Sleep Apnoea and Type 2 Diabetes, International Diabetes Federation, Brussels, Belguim, 2008. 


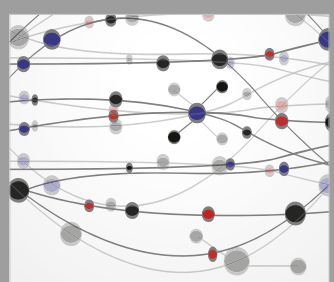

The Scientific World Journal
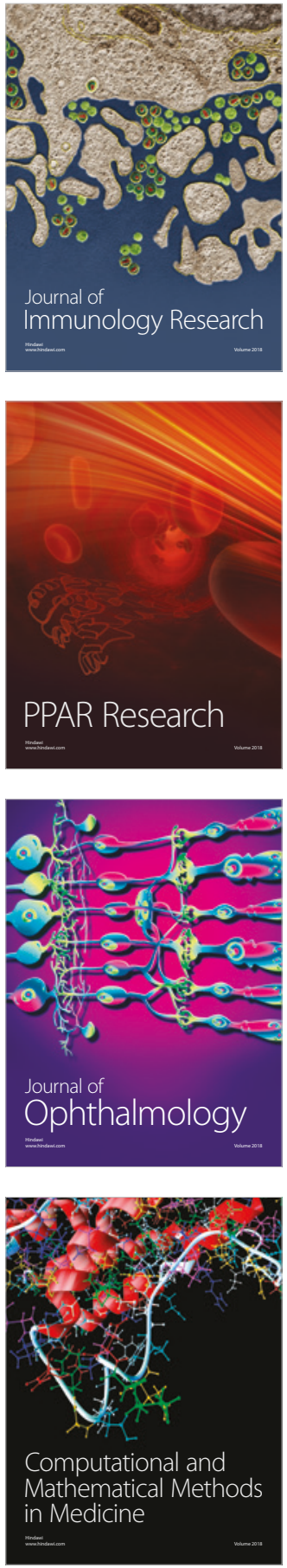

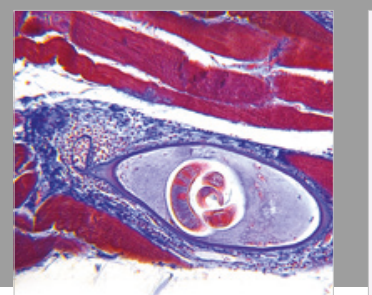

Gastroenterology Research and Practice

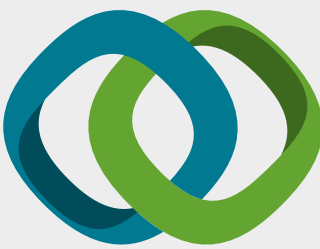

\section{Hindawi}

Submit your manuscripts at

www.hindawi.com
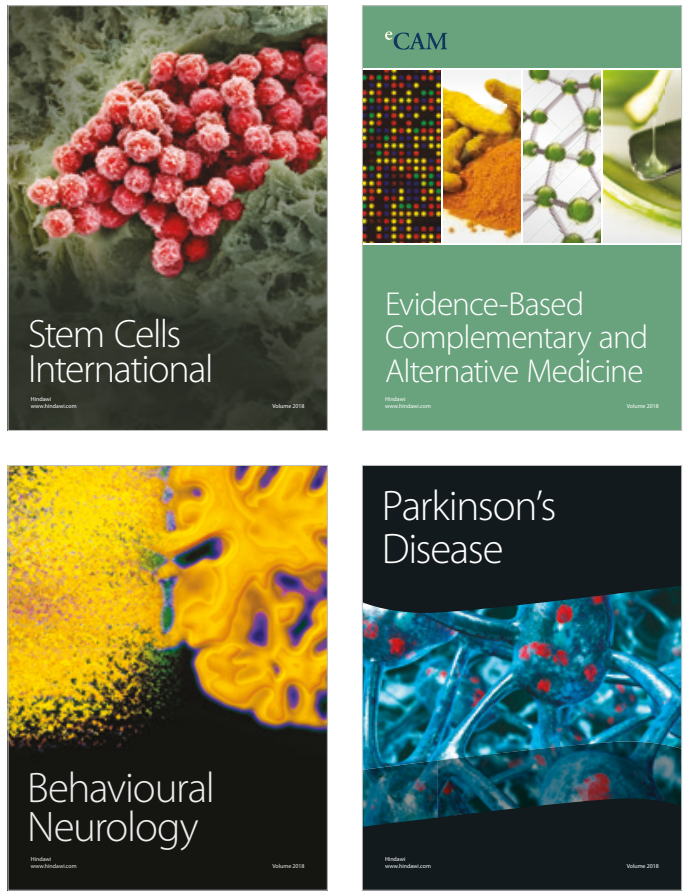

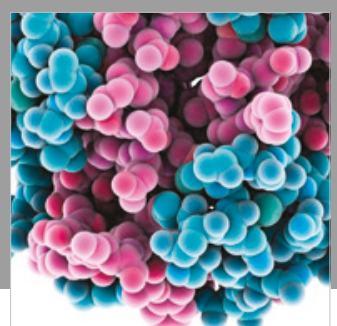

ournal of

Diabetes Research

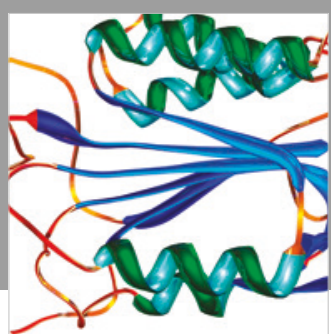

Disease Markers
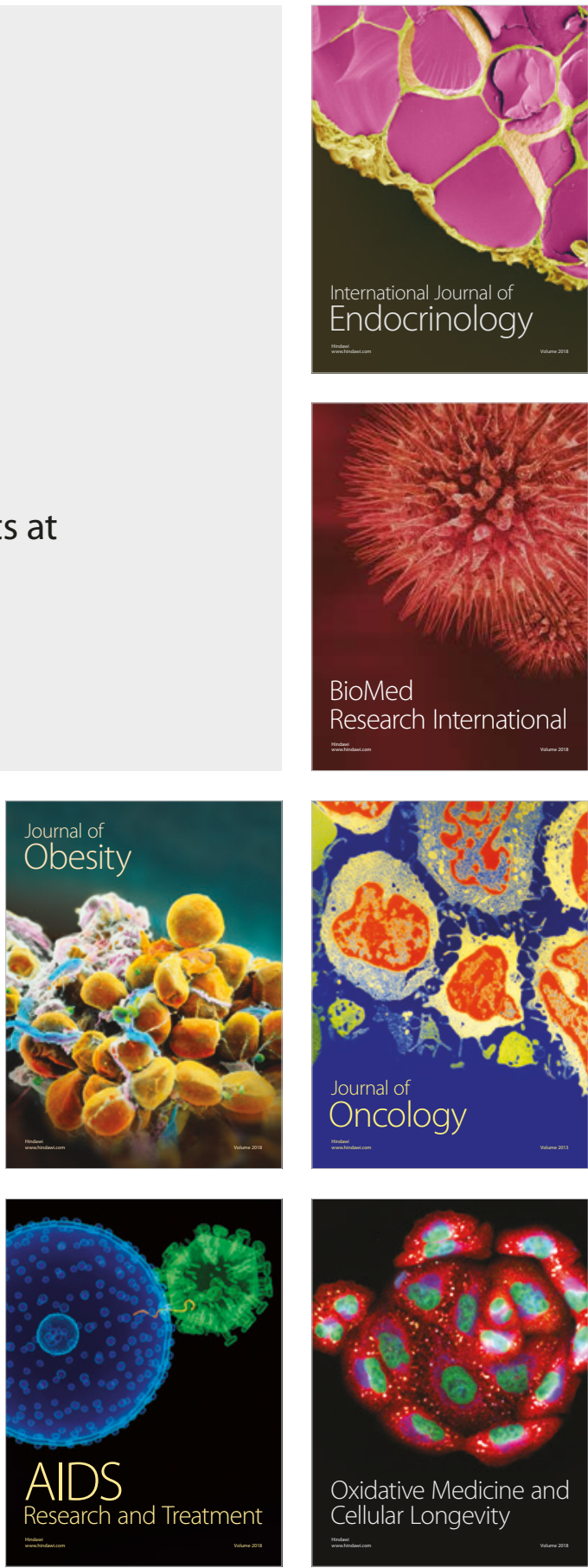